\title{
Balloon enteroscopy-assisted biliary drainage using a diathermic dilator followed by placement of a novel ultra-slim metallic stent
}

Endoscopic management of malignant biliary stricture after hepaticojejunostomy is challenging. Although balloon enteroscopy-assisted endoscopic retrograde cholangiopancreatography (ERCP) has been developed, dedicated devices are still insufficient. We present a case of successful enteroscopic dilation using a wire-guided diathermic dilator [1-5], and placement of a novel, ultra-slim, uncovered, self-expandable, metallic stent (USEMS) for the treatment of a severe malignant stricture of the perihilar bile duct after hepaticojejunostomy.

A 52-year-old man with obstructive jaundice was referred to our department. He had undergone pancreaticoduodenectomy with hepaticojejunostomy for pancreatic head cancer 27 months earlier. He had received 6 months of chemotherapy for multiple liver metastases, but this treatment was suspended because of multiple liver abscesses, which developed 1 month before we saw him.

Computed tomography revealed intrahepatic bile duct dilation, perihilar biliary mass, and ascites. Double-balloon enteroscopy (El-530B; Fujifilm Corp., Tokyo, Japan) was selected for biliary drainage. Double-balloon enteroscopy showed jejunal invasion of recurrent tumor involving the hepaticojejunal anas-

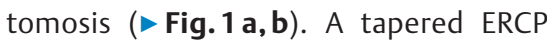
catheter and a 0.025-inch guidewire were passed through the stricture of the hepaticojejunal anastomosis ( $\mathbf{F i g} \mathbf{1} \mathbf{b}$ ). ERCP showed perihilar biliary stricture ( $\triangleright$ Fig.2a, $\triangleright$ Video 1), which could not be passed by the tapered ERCP catheter ( video 1).

The severe stricture was dilated successfully with a wire-guided diathermic dilator (Cysto-Gastro-Set, 6Fr, length $180 \mathrm{~cm}$; Endo-Flex, Voerde, Germany) ( $\triangleright$ Fig.2b, $>$ Video 1). Total dilation time was 3 seconds for the hepaticojejunal anastomosis and 13 seconds for the
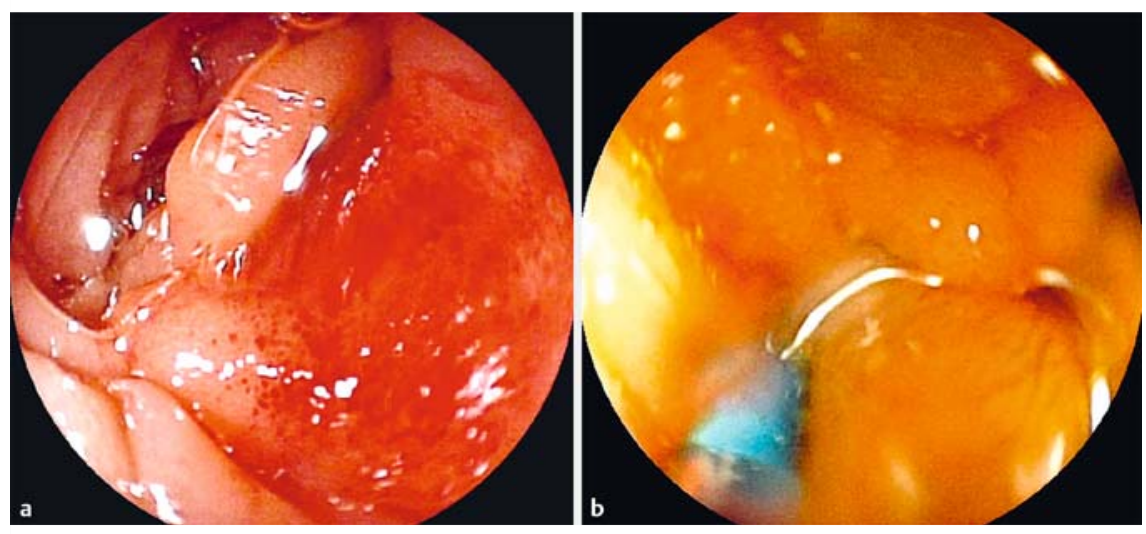

- Fig. 1 Endoscopic images. a Jejunal invasion. b Edematous mucosa around the hepaticojejunal anastomosis.

perihilar biliary stricture, respectively. A slim USEMS (Zilver 635, 6.0 Fr delivery system, $10 \mathrm{~mm}$ diameter, $200 \mathrm{~cm}$ long; Cook Japan, Tokyo, Japan) failed to pass the stricture. However, a novel ultra-slim USEMS (BileRush Selective, 5.7 Fr delivery system, $10 \mathrm{~mm}$ diameter, $185 \mathrm{~cm}$ long; Piolax Medical Devices, Kanagawa, Japan) was successfully deployed, followed by placement of an additional USEMS, in a stent-in-stent fashion, with- out any complications ( $\triangleright$ Fig. 3 , \ Fig. 4, - Video 1).

In summary, using balloon enteroscopy, a wire-guided diathermic dilator and novel ultra-slim USEMS were useful in facilitating dilation and stenting for the treatment of severe malignant anastomotic and biliary strictures in a patient with altered anatomy.

Endoscopy_UCTN_Code_TTT_1AS_2AD

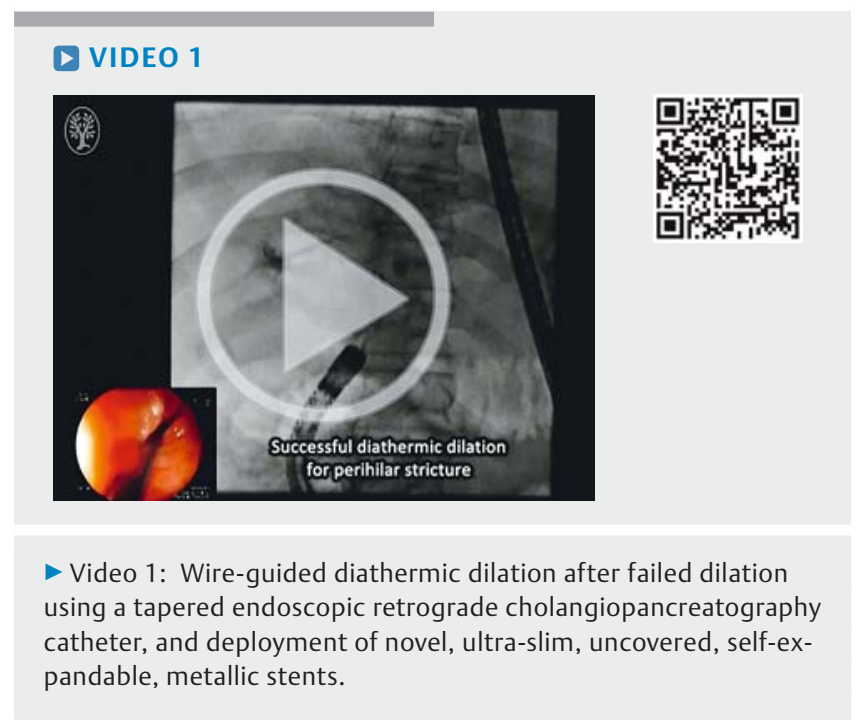



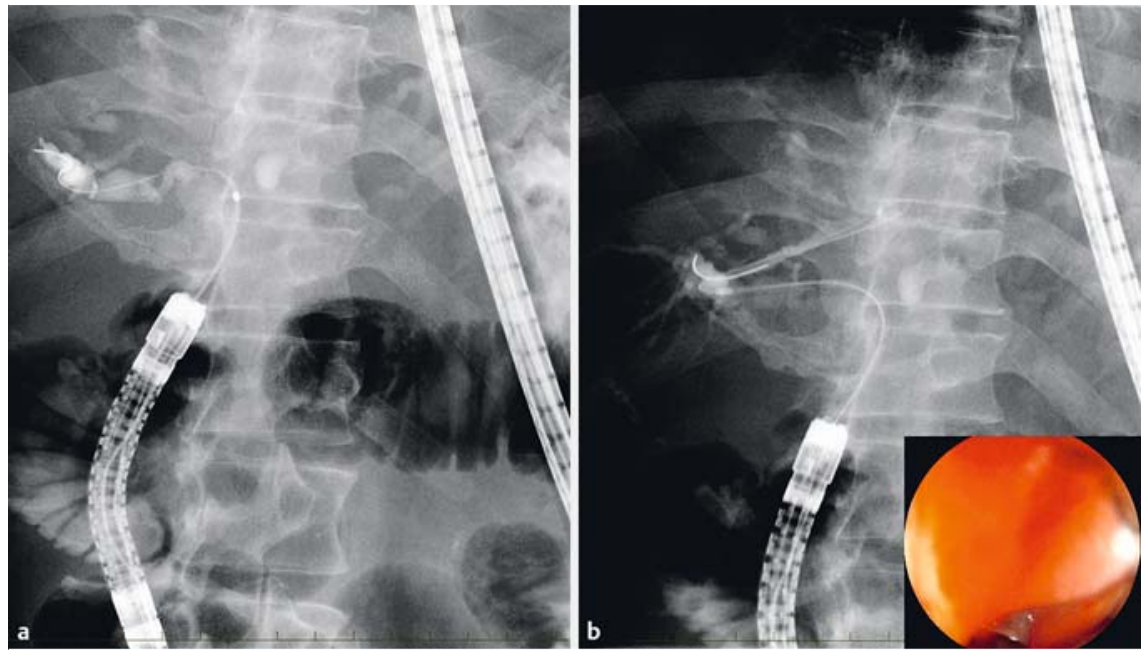

- Fig. 2 Radiographic images. a Perihilar biliary stricture. b Wire-guided diathermic dilator passing the perihilar bile duct stricture (inset: $6 \mathrm{Fr}$ wire-guided diathermic dilator insertion under endoscopic guidance).

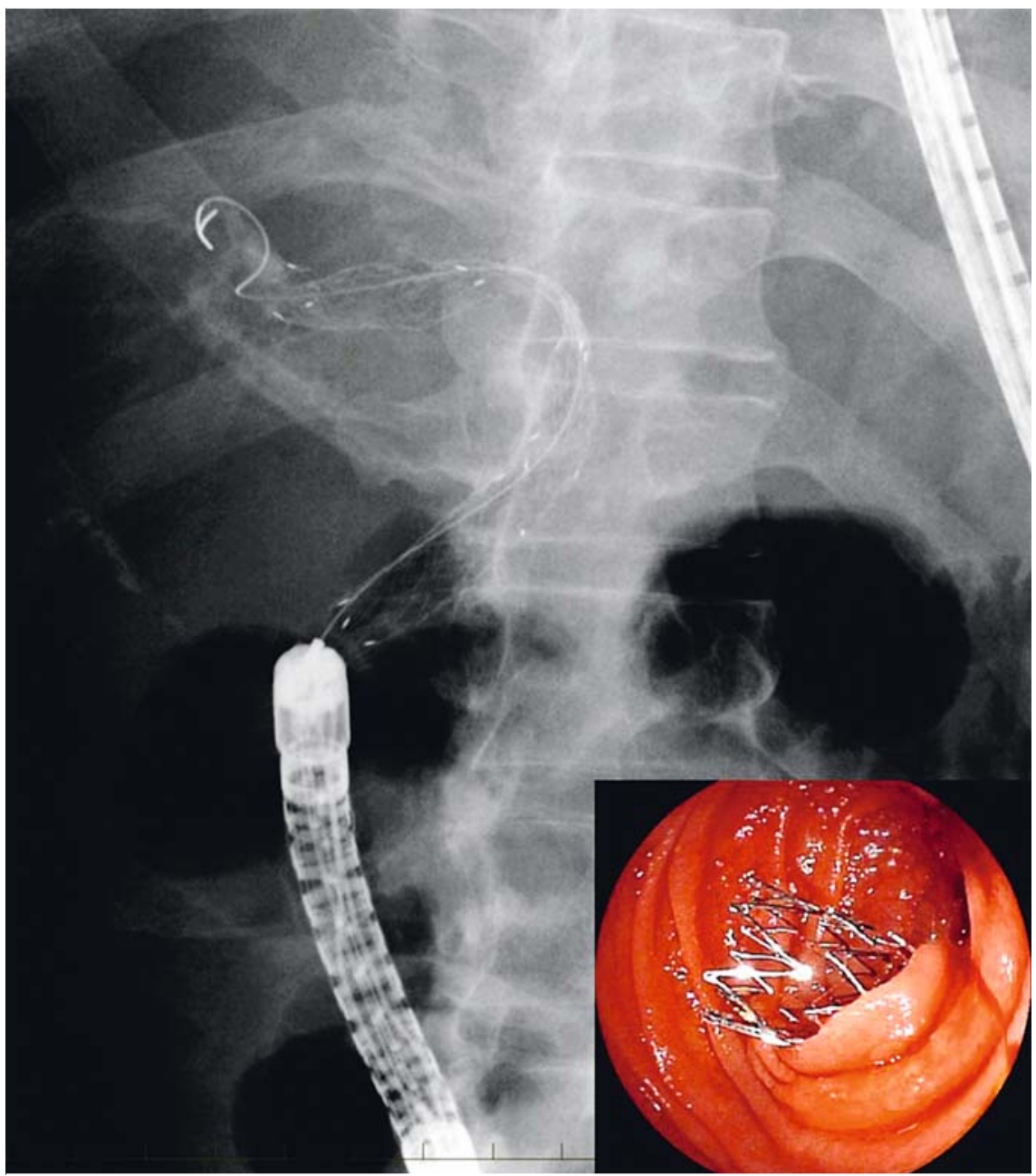

- Fig. 4 Radiograph showing deployed novel ultra-slim uncovered self-expandable metallic stent (inset: enteroscopic view)

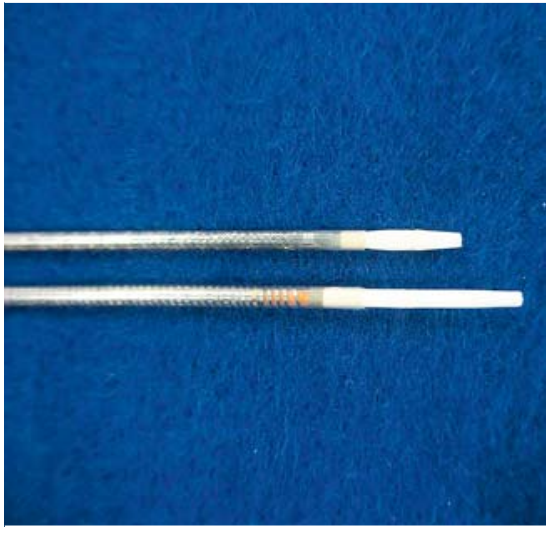

- Fig. 3 Comparison of a novel, ultraslim, uncovered, self-expandable, metallic stent (USEMS; BileRush Selective, 5.7 Fr delivery system, $10 \mathrm{~mm}$ diameter, $185 \mathrm{~cm}$ long; PIOLAX Medical Devices, Kanagawa, Japan) (above), and a slim USEMS (Zilver $635,6.0 \mathrm{Fr}$ delivery system, $10 \mathrm{~mm}$ diameter, $200 \mathrm{~cm}$ long; Cook Japan, Tokyo, Japan) (below).

\section{Competing interests}

Dr. Kawakami has collaborated with Piolax Medical Devices on the development of the BileRush Selective, and is a consultant and gives lectures for the company.

The Authors

Hiroshi Kawakami ${ }^{1,2}$, Yoshimasa Kubota ${ }^{1,2}$, Shinya Ashizuka ${ }^{2,3}$, Masaru Haraguchi' ${ }^{2,3}$, Ichiro Sannomiya ${ }^{2,3}$

1 Department of Gastroenterology and Hepatology, Faculty of Medicine, University of Miyazaki, Miyazaki, Japan

2 Center for Digestive Disease, University of Miyazaki Hospital, Miyazaki, Japan

3 Circulatory and Body Fluid Regulation, Faculty of Medicine, University of Miyazaki, Miyazaki, Japan

\section{Corresponding author}

\section{Hiroshi Kawakami, MD, PhD}

Department of Gastroenterology and Hepatology, Faculty of Medicine, University of Miyazaki, Center for Digestive Disease, University of Miyazaki Hospital, 5200, Kihara, Kiyotake Miyazaki 889-1692, Japan Fax: +81-985-859802 hiropon@med.miyazaki-u.ac.jp 


\section{References}

[1] Kawakami H, Kuwatani M, Eto K et al. Resolution of a refractory severe biliary stricture using a diathermic sheath. Endoscopy 2012; 44 (Suppl. 02): E119-120

[2] Kawakami H, Kuwatani M, Kawakubo K et al. Transpapillary dilation of refractory severe biliary stricture or main pancreatic duct by using a wire-guided diathermic dilator (with video). Gastrointest Endosc 2014; 79: 338 343

[3] Kawakami H, Kuwatani M, Sakamoto N. Endoscopic ultrasound-guided antegrade diathermic dilation followed by self-expandable metallic stent placement for anastomotic stricture after hepaticojejunostomy (with video). Dig Endosc 2014; 26: 121 - 122

[4] Kawakami H, Kuwatani M, Kawakubo K et al. Endoscopic ultrasound-guided antegrade diathermic dilation followed by self-expandable metal stent placement for malignant distal biliary stricture. Endoscopy 2014; 46 (Suppl. 01): E328-329

[5] Kawakami H, Abo D, Kawakubo K et al. Rendezvous biliary recanalization combining percutaneous and endoscopic techniques using a diathermic dilator for bile duct obstruction. Endoscopy 2014; 46 (Suppl. 01): E460 - 461

\section{Bibliography}

DOI http://dx.doi.org/10.1055/s-0042-124499 Endoscopy 2017; 49: E78-E80

(c) Georg Thieme Verlag KG

Stuttgart · New York

ISSN 0013-726X 